Algebraic $\&$ Geometric Topology

Volume 3 (2003) 557-568

Published: 19 June 2003

ATG

\title{
A geometric interpretation of Milnor's triple linking numbers
}

\author{
Blake Mellor \\ PAUl MELVIN
}

\begin{abstract}
Milnor's triple linking numbers of a link in the 3-sphere are interpreted geometrically in terms of the pattern of intersections of the Seifert surfaces of the components of the link. This generalizes the well known formula as an algebraic count of triple points when the pairwise linking numbers vanish.
\end{abstract}

AMS Classification 57M25; 57M27

Keywords $\bar{\mu}$-invariants, Seifert surfaces, link homotopy

\section{Introduction}

Milnor's higher order linking numbers (or mu-bar invariants) $\bar{\mu}_{\mathrm{I}}$ of an oriented link $L=L_{1} \cup \cdots \cup L_{\ell}$ in the 3 -sphere are a measure of how deep the longitudes $l_{i}$ of the components $L_{i}$ lie in the lower central series of the link group $\pi=$ $\pi_{1}\left(S^{3}-L\right)$. They are defined as follows [4]:

Any diagram of $L$ gives rise to a Wirtinger presentation of $\pi$ whose generators are meridians of the link, one for each arc in the diagram. If one works modulo any term $\pi^{n}$ in the lower central series of $\pi$ (where $\pi^{1}=\pi$ and $\pi^{n}=\left[\pi, \pi^{n-1}\right]$ ) then only one meridian $m_{i}$ is needed from each component $L_{i}$. In particular the longitude $l_{i}$ can be written $\bmod \pi^{n}$ as a word $l_{i}^{n}$ in these preferred meridians, i.e. as an element of the free group $F=F\left(m_{1}, \ldots, m_{\ell}\right)$. Now each element in $F$ can be viewed as a unit in the ring $A$ of power series in the noncommuting variables $h_{1}, \ldots, h_{\ell}$ by substituting $1+h_{i}$ for $m_{i}$ and $1-h_{i}+h_{i}^{2}-+\cdots$ for $m_{i}^{-1}$; this is its Magnus expansion [3] (which embeds $F$ in $A$ ). For any sequence $\mathrm{I}=i_{1} \cdots i_{r}$ of integers between 1 and $\ell$, let $\mu_{\mathrm{I}}$ denote the coefficient of $h_{i_{1}} \cdots h_{i_{r-1}}$ in the Magnus expansion of $l_{i_{r}}^{n}$ for large $n$. It is easily shown that $\mu_{\mathrm{I}}$ is independent of $n$ for $n \geq r$, but unfortunately it does depend on the original choice of meridians. If however one reduces modulo the greatest 
common divisor $\delta_{\mathrm{I}}$ of the lower order coefficients $\mu_{\mathrm{J}}$ (for all proper subsequences $\mathrm{J}$ of I) then one obtains Milnor's linking numbers

$$
\bar{\mu}_{\mathrm{I}}=\mu_{\mathrm{I}}\left(\bmod \delta_{\mathrm{I}}\right) \in \mathbb{Z} / \delta_{\mathrm{I}} \mathbb{Z}
$$

They are invariants of $L$ (with a given ordering of its components) up to link concordance, and in fact up to link homotopy if the indices $i_{1}, \ldots, i_{r}$ are distinct. Note that in general $\bar{\mu}_{\mathrm{I}}$ depends on the ordering of the indices in I, although it is symmetric in these indices for $r=2$ (indeed $\mu_{i j}=\bar{\mu}_{i j}$ is the usual pairwise linking number) and antisymmetric for $r=3$.

The computation of $\bar{\mu}_{\mathrm{I}}$ from this point of view is tedious and unenlightening, especially for large $r$. As a warm-up consider the pairwise linking number $\bar{\mu}_{i j}$, which is the coefficient of $h_{i}$ in the Magnus expansion of $l_{j}^{2}$. To compute $\bar{\mu}_{i j}$, write the word for the longitude $l_{j}$ as a product of generators in the Wirtinger presentation, each of which is a conjugate $w m_{p} w^{-1}$ of its associated (preferred) meridian $m_{p}$. Working mod the commutator subgroup $\pi^{2}$, this conjugate can be replaced by just $m_{p}$, giving a word $u_{j}$ in $m_{1}, \ldots, m_{\ell}$. Then

$$
\bar{\mu}_{i j}=\varepsilon_{i}\left(u_{j}\right)
$$

where $\varepsilon_{i}\left(u_{j}\right)$ is the sum of the exponents of $m_{i}$ in $u_{j}$ (cf. $[2, \mathrm{p} 8]$ ).

Now consider the triple linking number $\bar{\mu}_{i j k}$ for distinct $i, j, k$, which is the coefficient of $h_{i} h_{j}$ in the Magnus expansion of $l_{k}^{3}$, reduced modulo the greatest common divisor $\delta=\delta_{i j k}$ of the pairwise linking numbers of $L_{i}, L_{j}$ and $L_{k}$. As before, write the word for the longitude $l_{k}$ as a product of generators in the Wirtinger presentation, and then express each generator as a conjugate $w m_{p} w^{-1}$ of its associated meridian. Working mod $\pi^{3}$, each generator in the conjugating word $w$ can be replaced by its associated meridian, giving the word $l_{k}^{3}$ in $m_{1}, \ldots, m_{\ell}$. Since we are only interested in the coefficient of $h_{i} h_{j}$ in the Magnus expansion of this word, any occurrence of meridians other than $m_{i}$ and $m_{j}$ can be ignored. Thus we are left with a word $u_{k}$ in $m_{i}$ and $m_{j}$. Now

$$
\bar{\mu}_{i j k}=\varepsilon_{i j}\left(u_{k}\right)(\bmod \delta)
$$

where $\varepsilon_{i j}\left(u_{k}\right)$ denotes the sum of the signed occurences of $m_{i} m_{j}$ in the word $u_{k}$ : each $\cdots m_{i}^{r} \cdots m_{j}^{s} \cdots$ in $u_{k}$, where $r, s= \pm 1$, contributes $r s$ to $\varepsilon_{i j}\left(u_{k}\right)$ (see $[2, \operatorname{pp} 38,142])$. (In fact $\bar{\mu}_{i j k}$ can be computed as $\varepsilon_{i j}(u)$ for any word $u$ representing $l_{k} \bmod \pi^{3}$.) 
The purpose of this note is to reinterpret this process for computing the triple linking numbers in terms of Seifert surfaces of the link components and their intersections. This generalizes work of Cochran [1] for links with trivial pairwise linking numbers (also see Turaev [5]).

Remarks It is worth noting a few properties of $\varepsilon_{i}$ and $\varepsilon_{i j}$. Let $u$ and $v$ be words in the meridians $m_{1}, \ldots, m_{\ell}$. Then

(1) $\varepsilon_{i}(u v)=\varepsilon_{i}(u)+\varepsilon_{i}(v)$

(2) $\quad \varepsilon_{i j}(u v)=\varepsilon_{i j}(u)+\varepsilon_{i j}(v)+\varepsilon_{i}(u) \varepsilon_{j}(v)$

(3) $\varepsilon_{i j}(u)+\varepsilon_{j i}(u)=\varepsilon_{i}(u) \varepsilon_{j}(u)$

(cf. [2, Lemma 4.2.7]). The third equation identifies two ways of counting (with sign) all pairs of an occurrence of $m_{i}$ and an occurrence of $m_{j}$ (in either order).

The authors gratefully acknowledge the hospitality of the Mathematics Institute at the University of Warwick during the Geometry/Topology Workshop in July 2000, where this work was initiated.

\section{The formula for $\bar{\mu}_{i j k}$}

Fix three components $L_{i}, L_{j}, L_{k}$ of $L$, with $\delta$ the greatest common divisor of their pairwise linking numbers, and choose any associated oriented Seifert surfaces $F_{i}, F_{j}, F_{k}$ in general position. Set $F=F_{i} \cup F_{j} \cup F_{k}$. It will be shown below that $\bar{\mu}_{i j k}$ is the difference of two geometric invariants, $t_{i j k}(F) \in \mathbb{Z}$ and $m_{i j k}(F) \in \mathbb{Z} / \delta \mathbb{Z}$, defined as follows.

The intersection $F_{i} \cap F_{j} \cap F_{k}$ consists of isolated triple points, each of which is given a sign according to the orientations and ordering of the surfaces: the sign is positive if and only if the ordered basis of normal vectors to $F_{i}, F_{j}, F_{k}$ at the triple point agrees with the standard orientation on $S^{3}$. Let

$$
t_{i j k}(F)=\# F_{i} \cap F_{j} \cap F_{k},
$$

the total number of triple points, counted with sign. This integer is clearly invariant under isotopies of the individual Seifert surfaces which maintain their mutual general position, and is antisymmetric in the indices.

Similarly any pairwise intersection of the Seifert surfaces is a union of circles, clasps and ribbons (see Figure 1). 

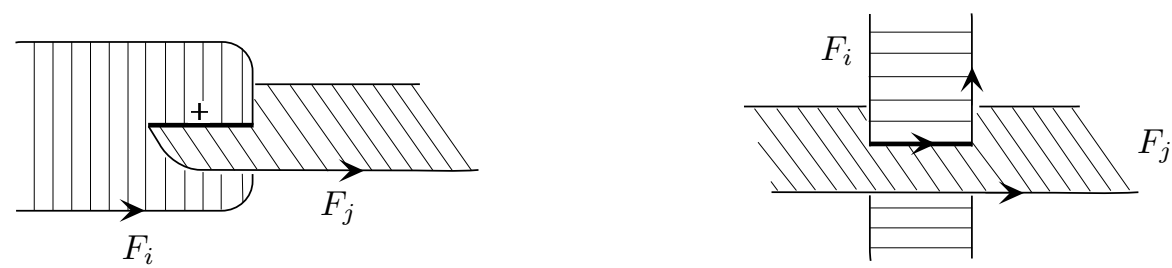

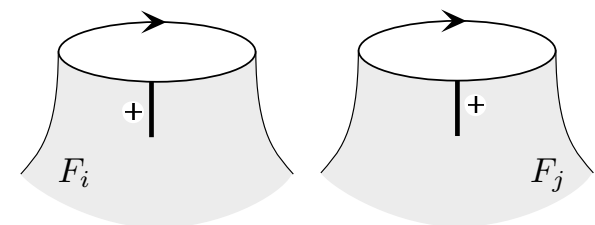

(a) clasp

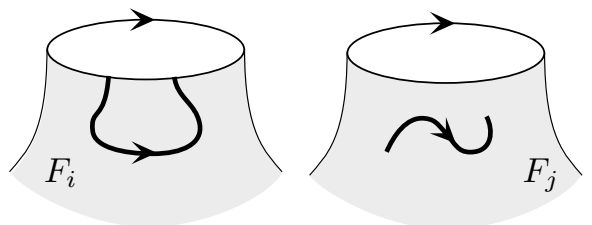

(b) ribbon

Figure 1: Intersections of Seifert surfaces

The endpoints of the clasps and ribbons are oriented in a natural way since they are intersection points of one oriented surface with the oriented boundary of another. Observe that the two endpoints of any clasp always have the same orientation, while the endpoints of a ribbon are oppositely oriented, and so these orientations can be specified by a single sign for each clasp and an orientation on each ribbon arc (from negative to positive) as shown in the figure.

Now choose a basepoint on each link component (this is equivalent to choosing the meridians in the definition of Milnor's invariant). Starting at the basepoint of $L_{i}$, read off a word $w_{i}$ in $j$ and $k$ by proceeding around $L_{i}$ : each double point in $L_{i} \cap F_{j}$ of sign $\varepsilon$ contributes $j^{\varepsilon}$, and similarly for $k$. (Here and below we simply use the indices $i, j, k$ to denote the corresponding meridians $m_{i}, m_{j}, m_{k}$.) Similarly define words $w_{j}$ and $w_{k}$. Let $e_{p q r}(F)$ denote the coefficient of $h_{p} h_{q}$ in the Magnus expansion of $w_{r}$, i.e. $e_{p q r}(F)=\varepsilon_{p q}\left(w_{r}\right)$, and set

$$
m_{i j k}(F)=e_{i j k}(F)+e_{j k i}(F)+e_{k i j}(F),
$$

the sum of the $e_{i j k}$ 's over cyclic permutation of the indices.

Example The Borromean rings are shown in Figure 2, bounding three disks with two clasps and one ribbon. If the basepoints are chosen near the labels, we compute $w_{i}=1, w_{j}=k k^{-1}=1$, and $w_{k}=i j i^{-1} j^{-1}$. Thus $e_{i j k}=1$ and $e_{j k i}=e_{k i j}=0$, so $m_{i j k}=1$. Note that the clasps can be eliminated, at the expense of introducing a triple point, by sliding the disk $F_{j}$ to the left so that its boundary encircles $F_{i}$ and pierces $F_{k}$ (cf. the theorem below). 

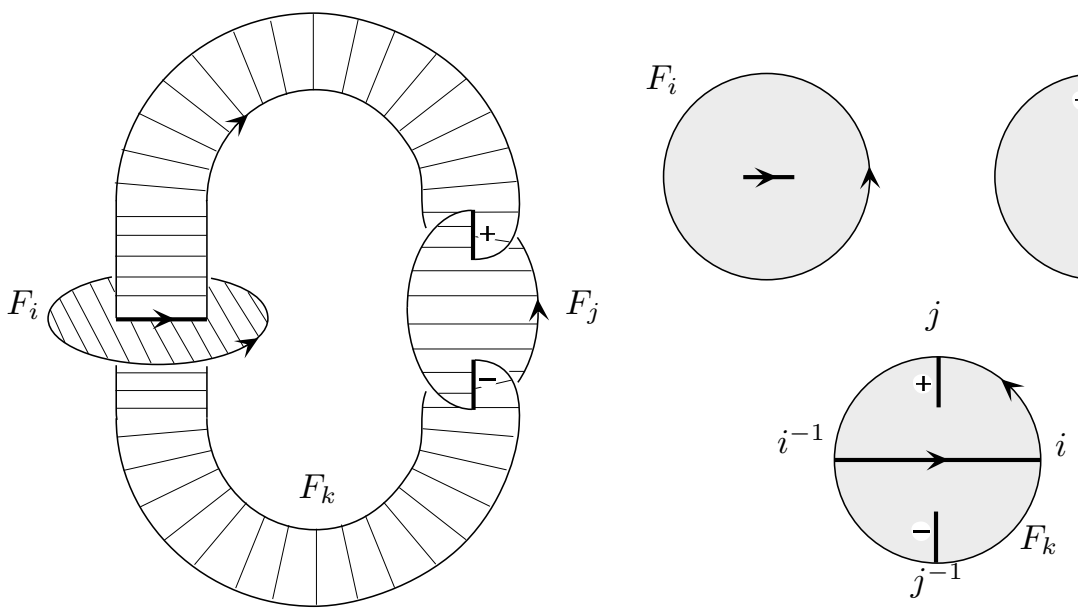

Figure 2: Computation of $m_{i j k}$

Remarks (1) Choosing a different basepoint changes $e_{i j k}$, and hence $m_{i j k}$, by some multiple of the linking numbers, so $m_{i j k}$ (like $\bar{\mu}_{i j k}$ ) is well defined modulo $\delta$. To see this, note that a basepoint change induces a cyclic permutation of $w_{k}$, which reduces the value of $e_{i j k}$ by $\varepsilon_{i j}(u v)-\varepsilon_{i j}(v u)$ for some factorization $w_{k}=u v$. But (using Remarks 1 and 2 at the end of the previous section)

$$
\begin{aligned}
\varepsilon_{i j}(u v)-\varepsilon_{i j}(v u) & =\varepsilon_{i}(u) \varepsilon_{j}(v)-\varepsilon_{i}(v) \varepsilon_{j}(u) \\
& =\left(\varepsilon_{i}(u)+\varepsilon_{i}(v)\right) \varepsilon_{j}(v)-\varepsilon_{i}(v)\left(\varepsilon_{j}(u)+\varepsilon_{j}(v)\right) \\
& =\varepsilon_{i}(u v) \varepsilon_{j}(v)-\varepsilon_{i}(v) \varepsilon_{j}(u v)=\mu_{i k} \varepsilon_{j}(v)+\varepsilon_{i}(v) \mu_{j k} .
\end{aligned}
$$

where $\mu_{p q}=\operatorname{lk}\left(L_{p}, L_{q}\right)$, so this difference is trivial $\bmod \delta$.

(2) The invariants $e_{i j k}$ are antisymmetric in $i$ and $j$. Indeed $e_{i j k}+e_{j i k}=$ $\varepsilon_{i j}\left(w_{k}\right)+\varepsilon_{j i}\left(w_{k}\right)=\varepsilon_{i}\left(w_{k}\right) \varepsilon_{j}\left(w_{k}\right)=\mu_{i k} \mu_{j k} \equiv 0(\bmod \delta)$, by Remark 3 in $\S 1$. It follows that $m_{i j k}$ is totally antisymmetric.

Our goal is to prove the following result, where as usual $\delta$ denotes the greatest common divisor of the pairwise linking numbers of $L_{i}, L_{j}$ and $L_{k}$.

Theorem (1) For any choice of Seifert surfaces $F$,

$$
\bar{\mu}_{i j k} \equiv m_{i j k}(F)-t_{i j k}(F) \quad(\bmod \delta) .
$$

(2) There is a choice of $F$ such that $t_{i j k}(F)=0$, so $\bar{\mu}_{i j k} \equiv m_{i j k}(F)(\bmod \delta)$.

(3) There is a choice of $F$ such that $m_{i j k}(F) \equiv 0$, so $\bar{\mu}_{i j k} \equiv-t_{i j k}(F)(\bmod \delta)$. 
The familiar fact that the Milnor invariants $\bar{\mu}_{i j k}$ are antisymmetric in the indices follows immediately from the theorem and the preceding remark. We also recover Cochran's result that $\bar{\mu}_{i j k}=-t_{i j k}(F)$ if the boundary of $F$ is disjoint from the double point set (which is only possible if the pairwise linking numbers vanish).

The idea of the proof is as follows. We will define two special types of of Seifert surfaces $F$ and show that $m_{i j k}(F) \equiv \bar{\mu}_{i j k}$ and $t_{i j k}(F)=0$ for those of type 1, while $m_{i j k}(F) \equiv 0$ for those of type 2 . We will then describe a set of finger moves that can transform an arbitrary $F$ into either reduced type, and that preserve $m_{i j k}-t_{i j k}(\bmod \delta)$. The theorem follows.

\section{Proof of the theorem}

A generic family $F=F_{i} \cup F_{j} \cup F_{k}$ of Seifert surfaces for the link $L_{i} \cup L_{j} \cup L_{k}$ will be called reduced if it is of one of the following types:

TyPe 1: (DISJOINT Clasps) The only double curves are disjoint clasps.

Type 2: (Boundary ordered) Around the boundary of any one of the three surfaces, all intersections with either of the other two are adjacent, except possibly for cancelling pairs (i.e. adjacent intersections of opposite sign with the second surface that may occur between intersections with the third).

If $F$ is a family of type 1 , then $t_{i j k}(F)=0$ (since there are no triple points at all) and $m_{i j k}(F)$ is just the $\bar{\mu}$-invariant (as is shown in the lemma below). If $F$ is of type 2 , then by definition the word $w_{k}$ (see $\S 2$ ) is of the form $m_{i}^{p} m_{j}^{q}$ for an appropriate choice of basepoint on $L_{k}$, where $p=\mu_{i k}$ and $q=\mu_{j k}$ (the linking numbers of $L_{i}$ and $L_{j}$ with $L_{k}$ ) and similarly for $w_{i}$ and $w_{j}$. Thus $e_{i j k}(F)=\varepsilon_{i j}\left(w_{k}\right)=p q \equiv 0(\bmod \delta)$, and so $m_{i j k}(F) \equiv 0$. It will follow from the theorem that in this case $t_{i j k}(F)$ reduces $(\bmod \delta)$ to the negative of the $\bar{\mu}$-invariant.

Lemma 1 If $F$ is of type 1 , then $m_{i j k}(F) \equiv \bar{\mu}_{i j k}$.

Proof By the classification of surfaces, each component of $F$ can be viewed as a 2-dimensional handlebody, i.e. a disk (0-handle) with bands (1-handles) attached, while the clasps between any two components can be viewed as pairs of feelers, i.e. bands attached at one end to their respective disks and clasped with each other at the other end. 
Of course the bands and feelers may be highly linked and twisted, so consider the effect of a band pass (moving one such through another or through itself). Clearly $m_{i j k}(F)$ is unchanged, since the pattern of double curves is unaltered, and $\bar{\mu}_{i j k}$ is certainly unchanged under self-passes and passes between bands from the same surface component, since it is a link homotopy invariant. In fact $\bar{\mu}_{i j k}$ is also invariant under passes between bands from distinct components. To see this, it suffices (using the antisymmetry of $\bar{\mu}$ ) to consider a pass between bands on $F_{j}$ and $F_{k}$ as shown below.
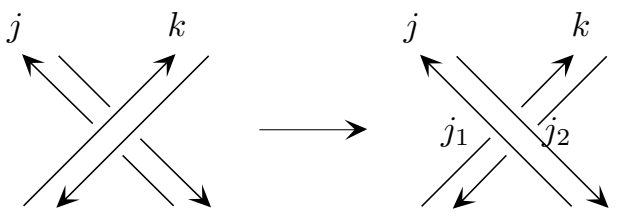

If the longitude of $L_{k}$ on the left is the word $u v$ (where $u$ starts at the lower left point) then on the right it is ${ }^{\left(j_{1}^{-1} j_{2}\right)} u v$ (where ${ }^{a} b$ denotes the conjugate $a b a^{-1}$ ) for suitable conjugates $j_{1}, j_{2}$ of the meridian $j$ of $L_{j}$. But

$$
\varepsilon_{i j}\left({ }^{\left(j_{1}^{-1} j_{2}\right)} u v\right)=\varepsilon_{i j}\left({ }^{\left(j^{-1} j\right)} u v\right)=\varepsilon_{i j}(u v),
$$

since in general $\varepsilon_{i j}\left(\cdots{ }^{(a b)} c \cdots\right)=\varepsilon_{i j}\left(\cdots{ }^{b} c \cdots\right)$, and so $\bar{\mu}_{i j k}$ remains unchanged.

Using band passes, the three surfaces can be "disentangled", except for the clasps. In particular, they can be placed so that their disks and bands lie near the vertices of a triangle whose edges are formed by the feelers reaching out to clasp each other (see Figure 3 for an example). Furthermore, it can be arranged that the only link crossings aside from the clasps (under the projection to the plane of the triangle) are between feelers from the same surface component, or between bands from the same component. In fact the latter can be changed at will, since self-crossings of a component do not contribute to $m_{i j k}$ or $\bar{\mu}_{i j k}$. Thus we may assume that each Seifert surface consists only of a disk with some feelers, as shown in the figure.

Now $\bar{\mu}_{i j k}=\varepsilon_{i j}\left(u_{k}\right)$, where $u_{k}$ is the word defined in $\S 1$ which represents the longitude $l_{k}$. For the case at hand, $u_{k}$ is clearly a product of conjugates of $i$ and $j$ and their inverses. In particular, each positive clasp of $F_{i}$ with $F_{k}$ contributes a conjugate $j^{-p} i j^{p}$, where $p$ is the number of clasps of $F_{i}$ with $F_{j}$ (counted with sign) which precede the given clasp along $L_{i}$. (Actually the conjugating element is a word in $i$ and $j$ with total exponent $p$ in $j$, but the $i$ 's can be ignored when computing $\varepsilon_{i j}$.) Likewise each negative clasp contributes $j^{-p} i^{-1} j^{p}$, and so the total contribution from the clasps between $F_{i}$ and $F_{k}$ is $e_{j k i}$ (since $\left.\varepsilon_{i j}\left(j^{-p} i^{ \pm 1} j^{p}\right)= \pm p\right)$. A similar argument shows that the 
contribution from the clasps between $F_{j}$ and $F_{k}$ is $-e_{i k j}$ (the minus sign arising since $\left.\varepsilon_{i j}\left(i^{-p} j^{ \pm 1} i^{p}\right)=\mp p\right)$. So the total internal contributions from these factors is $e_{j k i}-e_{i k j}=e_{j k i}+e_{k i j}$ (by Remark 2 in $\S 2$ ). The contribution from the order of the factors is $e_{i j k}$, so we conclude that $\bar{\mu}_{i j k}=e_{i j k}+e_{j k i}+e_{k i j} \equiv m_{i j k}$ as desired.

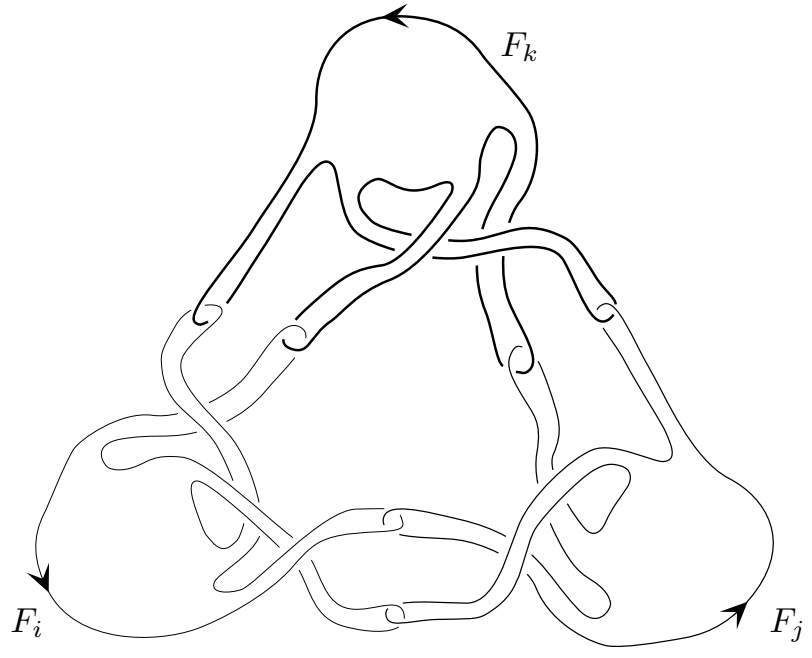

Figure 3: Disentangled surfaces

Remark There is a nice alternative proof that $\bar{\mu}_{i j k} \equiv m_{i j k}(F)$ for disentangled surfaces: One observes that the braiding of the feelers can be accomplished by "Borromean tangle" replacements - familiar from the theory of finite type invariants of 3-manifolds - one for each braid generator. One then shows that the invariants $\bar{\mu}_{i j k}$ and $m_{i j k}$ change by an equal amount $( \pm 1)$ under such a tangle replacement, and it is easy to check that they agree if there is no braiding.

We now define three "finger moves" on a generic family $F$ of Seifert surfaces. Each move involves pushing the interior of a small disk on one of the surfaces along a path in one of the other surfaces. It is understood that the path begins at the center of the disk, and that the center should be pushed slightly beyond the endpoint of the path.

First finger move : Push from a double point along a path to the boundary which contains no other double points.

Second finger move : Push from a triple point along a path to the boundary which lies in a double curve and contains no other triple points. 
Third Finger move : Push from a double point in the boundary to an adjacent double point along a path in the boundary.

These moves are illustrated in Figures 4, 5 and 6.

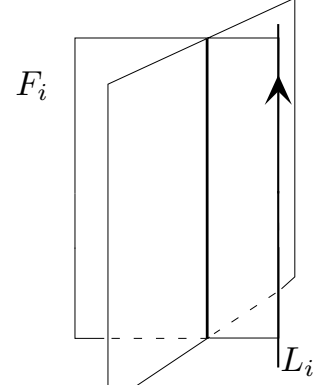

$F_{j}$
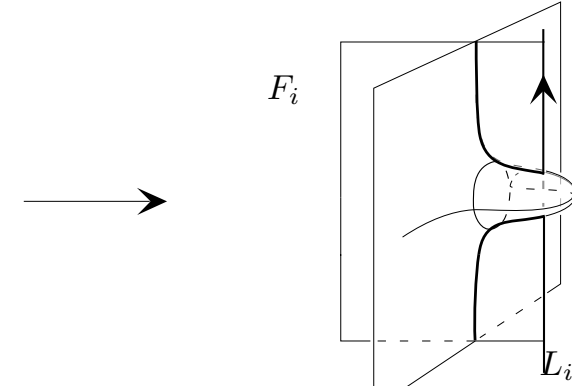

$F_{j}$

Figure 4: First finger move
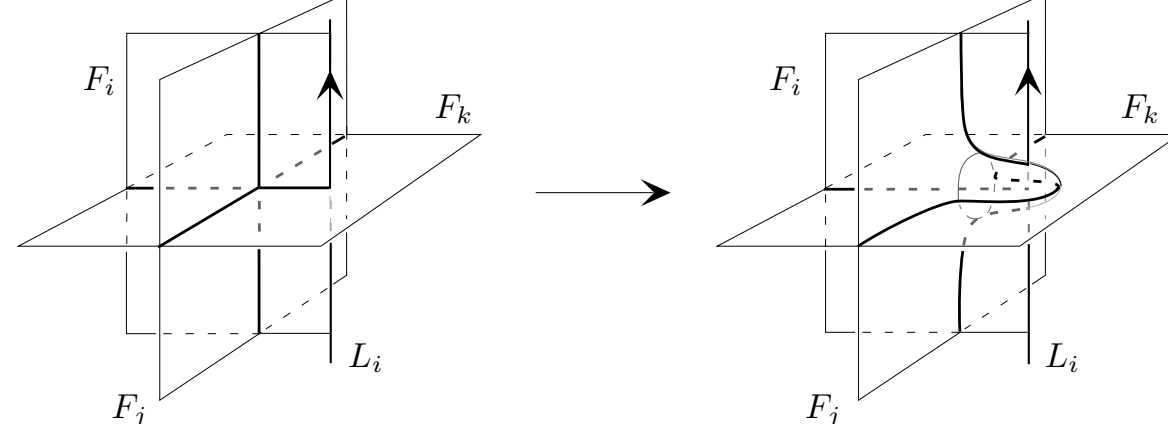

Figure 5: Second finger move
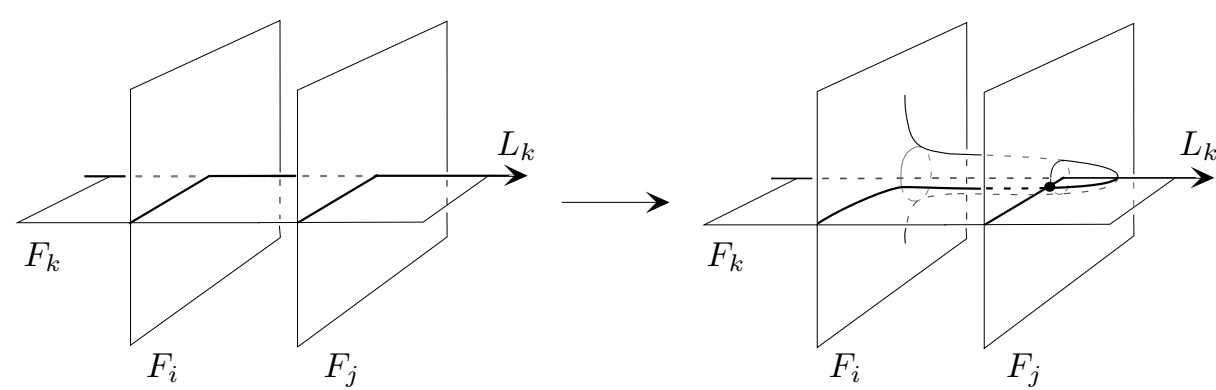

Figure 6: Third finger move

Algebraic 83 Geometric Topology, Volume 3 (2003) 
Lemma 2 The difference $m_{i j k}-t_{i j k}$ is invariant under all three finger moves.

Proof Each move is, to be exact, six possible moves, since the roles of the three surfaces can be permuted. We will consider only one of the six cases, the ones depicted in the figures, since the others can be proved similarly.

The first finger move (Figure 4) clearly does not change the number of triple points, so $t_{i j k}$ remains the same. Also, since the words $w_{j}$ and $w_{k}$ (around the boundaries of $F_{j}$ and $F_{k}$ ) are unchanged, so are $e_{i j k}$ and $e_{k i j}$. The word $w_{i}$ is only changed by adding a cancelling pair, so $e_{j k i}$ is unchanged. Therefore $m_{i j k}$ is fixed as well, and so is the difference $m_{i j k}-t_{i j k}$.

The second finger move changes the number of triple points by 1 . In the case shown in Figure 5, a positive triple point is removed, so $t_{i j k}$ decreases by 1 (the boundaries of the surfaces are oriented counterclockwise). The words $w_{j}$ and $w_{k}$ are unchanged; the word $w_{i}$ is modified by replacing one appearance of the letter $k$ by its conjugate $j^{-1} k j$. So $e_{i j k}$ and $e_{k i j}$ are unchanged, while $e_{j k i}$ decreases by 1 . The result is to decrease $m_{i j k}$ by 1 . Therefore the difference $m_{i j k}-t_{i j k}$ is unchanged.

The third finger move, as shown in Figure 6 , decreases $t_{i j k}$ by 1 by adding a negative triple point. The words $w_{i}$ and $w_{j}$ are unchanged, but $w_{k}$ is modified by replacing an adjacent pair of letters $i j$ by $j i$. So $e_{j k i}$ and $e_{k i j}$ remain fixed, while $e_{i j k}$ decreases by 1 . This means $m_{i j k}$ is reduced by 1 , and once again the difference $m_{i j k}-t_{i j k}$ is preserved.

The final step in the proof of the theorem is to show how to use finger moves to transform a generic family of Seifert surfaces into either one of the two reduced types. To achieve the first type, in which the intersections between the components are disjoint clasps, it is necessary to eliminate all circles, ribbons and triple points. We describe a procedure to accomplish this.

First, remove circles by finger moves of type 1 . Do this by following any path from the circle to the boundary of one of the surfaces in which it lies, breaking each double arc crossed by this path by a type 1 finger move. Note that this move never creates circles, so its repeated application eventually eliminates all the circles.

So now all triple points involve clasps and/or ribbons. We can inductively remove the triple points along each clasp or "long" ribbon (i.e. a double arc with both endpoints on the boundary of one surface) by using finger moves of type 2. Again, this move never creates circles, so we are left with a disjoint collection of clasps and ribbons. 
Finally, remove the ribbons by finger moves of type 1 on "short" ribbons (double arcs with both endpoints in the interior of the surface) along any path from the ribbon to the boundary which avoids other ribbons and clasps. Such a path will exist because there are no triple points or circles, so no short ribbon can be separated from the boundary. This leaves a collection of disjoint clasps, i.e. a reduced family of type 1 .

To obtain a reduced family of surfaces of the second type, begin with one of the first type, and then simply reorder the clasps as necessary via transpositions, using the third finger move. This completes the proof of the theorem.

Final Remarks (1) The theorem suggests a natural construction of a three component link with any prescribed pairwise linking numbers $p, q, r$ and any given triple linking number $m$ : Start with the unlink, bounding disjoint disks. Next add $p$ feelers between one pair of disks (as in Lemma 1), and similarly for $q$ and $r$ with the other pairs. Finally add $m$ Borromean tangles (band sums with the three components of the Borromean rings). Now apply the theorem, using the calculation in the Example in $\S 2$.

(2) If one allows nonorientable Seifert surfaces, then the geometric invariants $t_{i j k}$ and $m_{i j k}$ are still defined modulo 2. (Note that the mod 2 reduction of $\varepsilon_{i j}(w)$ depends only on the parity of the exponents of $i$ and $j$ in $w$.) Furthermore, the proof of the theorem carries over, without change, to show that $\bar{\mu}_{i j k} \equiv m_{i j k}-t_{i j k}(\bmod 2)$. Of course this is a vacuous statement unless the pairwise linking numbers are even.

(3) A geometric interpretation for the higher order Milnor invariants remains open. A formula for the first non-vanishing invariant is given by Cochran [1], using surfaces associated to certain derived links. It is natural to ask whether the ideas of this paper can be extended to the general case; the difficulty seems to lie in finding the right "higher-order surfaces." 


\section{References}

[1] T D Cochran, Derivatives of links: Milnor's concordance invariants and Massey's Products, Memoirs of the Amer. Math. Soc. 427 (1990)

[2] R A Fenn, Techniques of Geometric Topology, London Math. Soc. Lecture Note Series 57, Cambridge University Press (1983)

[3] W Magnus, A Karrass, D Solitar, Combinatorial Group Theory, Interscience Publishers (1966)

[4] J Milnor, Isotopy of links, in Algebraic Geometry and Topology (edited by R. Fox), Princeton University Press (1957) 280-306

[5] V Turaev, The Milnor invariants and Massey products, Zap. Nanc. Sem. LOMI Steklov 66 (1976) 189-210

Loyola Marymount University, One LMU Drive

Los Angeles, CA 90045, USA

and

Bryn Mawr College, $101 \mathrm{~N}$ merion Ave

Bryn Mawr, Pa 19010-2899, USA

Email: bmellor@lmu.edu, pmelvin@brynmawr.edu

Received: 7 June 2003

Algebraic 6 Geometric Topology, Volume 3 (2003) 